03,09

\title{
Спектроскопия резонансного возбуждения экситонной люминесценции твердых растворов GaSe-GaTe
}

\author{
() А.Н. Старухин, Д.К. Нельсон, Д.Л. Федоров, Д.К. Сюняев \\ Физико-технический институт им. А.Ф. Иофрфе РАН, \\ Санкт-Петербург, Россия \\ E-mail: a.starukhin@mail.ioffe.ru
}

(Поступила в Редакцию 23 декабря 2015 г.)

Исследованы спектры возбуждения люминесценции локализованных экситонов в твердых растворах $\mathrm{GaSe}_{0.85} \mathrm{Te}_{0.15}$ при $T=2 \mathrm{~K}$. Показано, что в спектрах возбуждения экситонов с энергией локализации $\varepsilon>10 \mathrm{mV}$ возникает дополнительный максимум $M_{E}$, расположенный с низкоэнергетической стороны от максимума, соответствующего полосе поглощения свободного экситона с $n=1$. Установлено, что сдвиг положения максимума $M_{E}$ в спектре возбуждения относительно энергии детектируемых фотонов увеличивается по мере уменьшения энергии детектируемых фотонов, то есть с увеличением энергии локализации экситонов. При резонансном возбуждении локализованных экситонов монохроматическим светом из области полосы экситонного излучения в спектре экситонной люминесценции с низкоэнергетической стороны от линии возбуждения также наблюдается максимум излучения $\left(M_{L}\right)$. Энергетическое расстояние между положением возбуждающей линии и положением максимума в спектре излучения увеличивается по мере уменьшения частоты возбуждающего света. Рассматриваются возможные механизм появления описанной структуры спектров возбуждения и экситонной люминесценции в $\mathrm{GaSe}_{0.85} \mathrm{Te}_{0.15}$. Сделан вывод, что максимумы $M_{E}$ в спектре возбуждения и $M_{L}$ в спектре излучения обусловлены электронно-колебательными переходами с рождением и аннигиляцией локализованных экситонов соответственно.

Работа выполнена при частичной поддержке РФФИ (проект № 13-02-00891).

DOI: 10.21883/FTT.2017.02.44039.481

\section{1. Введение}

Полупроводниковые твердые растворы представляют значительный интерес с фундаментальной и прикладной точек зрения [1]. Среди полупроводниковых твердых растворов, используемых в различных приложениях, значительное место занимают твердые растворы с изоэлектронным замещением. Твердые растворы изоэлектронного замещения сохраняют геометрический дальний порядок в расположении атомов и ряд их фундаментальных свойств может быть описан в рамках модели виртуального кристалла [2]. Однако в отличие от идеального кристалла флуктуации состава смешанного кристалла порождают флуктуационный электронный потенциал, ответственный за наличие границы подвижности для электронов и дырок и формирование хвостов плотности локализованных состояний в запрещенной зоне кристалла $[3,4]$. Кулоновское взаимодействие локализованных электронов (или дырок) с носителями заряда противоположного знака может приводить к формированию двухчастичных связанных состояний - локализованных экситонов [4-8].

При малых концентрациях одной из компонент раствора ее атомы можно рассматривать как изоэлектронные примеси. Характер электронного потенциала в области примеси и ее способность связать тот или иной носитель заряда связаны с различием электроотрицательностей замещаемого и примесного атомов $[9,10]$ : электроны атома большего радиуса находятся дальше от ядра и притягиваются к нему слабее, чем у изоэлектрон- ного атома меньшего радиуса. В частности, при замене атома селена теллуром в кристалле GaSe ион теллура в силу меньшей электроотрицательности является потенциальной ловушкой для дырки. В случае одиночной изоэлектронной примеси мощность потенциальной ямы может оказаться недостаточной для связывания носителя, но по мере увеличения концентрации замещающих атомов они формируют кластеры, в которых обеспечиваются условия для связывания носителя заряда $[4,7]$.

В условиях оптического возбуждения заселение состояний локализованных экситонов (ЛЭ) может происходить различными путями. Одним из них является описанный выше механизм последовательного захвата изоэлектронной примесью (или кластером „примесных“ атомов) носителей заряда противоположного знака. Другими возможными механизмами являются захват флюктуационным потенциалом экситона как целого и непосредственное рождение светом локализованного экситона с определенной энергией локализации („резонансное возбуждение“). Поскольку связанный короткодействующим потенциалом изоэлектронного атома носитель, например дырка, оказывается локализованным в малой области кристалла, резонансное оптическое возбуждение локализованного экситона существенно меняет зарядовое состояние этой области, а поле фотовозбужденной дырки может характеризоваться заметным градиентом на расстояниях порядка межатомного расстояния в кристалле, что должно приводить к изменениям равновесных положений атомов решетки в ближайшем 
окружении примеси [11], особенно в ионных кристаллах. В результате при резонансном оптическом возбуждении рождение локализованного экситона и его последующая аннигиляция могут проходить при различных конфигурациях ионов в области дефекта, что должно проводить к существенному Стоксову сдвигу максимумов в спектрах поглощения и излучения.

Целью настоящей работы явилось экспериментальное исследование этого эффекта в оптических спектрах ЛЭ в твердых растворах соединений $\mathrm{GaSe}_{1-x} \mathrm{Te}_{x}(x<0.3)$. При $x \leq 0.3$ соединения $\mathrm{GaSe}_{1-x} \mathrm{Te}_{x}$ кристаллизуются в решетку типа GaSe гексагональной симметрии $D_{3 h}$ [12] с изоэлектронным замещением части атомов селена теллуром. В рамках работы проведено сравнительное исследование оптических спектров фотолюминесценции и возбуждения фотолюминесценции ЛЭ в системе $\mathrm{GaSe}-\mathrm{GaTe}$ в условиях их резонансного возбуждения. Установлено, что положения максимумов в спектрах возбуждения и люминесценции характеризуются зеркальной симметрией относительно частоты резонансного перехода, и изучена зависимость эффекта от энергии локализации экситонов.

\section{2. Методика эксперимента}

Селенид и теллурид галлия относятся к группе слоистых кристаллов $A^{\mathrm{III}} B^{\mathrm{VI}}$. Твердые растворы $\mathrm{GaSe}_{1-x} \mathrm{Te}_{x}$ $(x=0.11,0.15)$ выращивались методом Бриджмена и специально не легировались. Образцы для исследований получались из слитков путем скалывания вдоль плоскости кристаллических слоев перпендикулярно оптической оси кристаллов. Возбуждение люминесценции кристаллов осуществлялось непрерывным излучением перестраиваемого по длине волны лазера на красителе. Плотность возбуждения составляла около $3 \mathrm{~W} / \mathrm{cm}^{2}$. Возбуждающий свет с энергией фотонов $h v_{\text {exc }}$ падал под малым углом к нормали $(\mathbf{n} \| z)$ к поверхности образца, излучение регистрировалось в направлении нормали, параллельно оптической оси кристалла $c$ (геометрия „на отражение“). Регистрация спектров осуществлялась с помощью дифракционного спектрометра, снабженного фотоприемным устройством на основе ПЗС-линейки. Для получения спектров возбуждения люминесценции измерялась интенсивность излучения на фиксированной частоте $h v_{d e t}$ в зависимости от частоты возбуждающего света $h v_{\text {exc }}$ при неизменной интенсивности возбуждения. Во время эксперимента образцы находились в жидком гелии при температуре $2 \mathrm{~K}$.

\section{3. Экспериментальные результаты}

Спектры люминесценции локализованных экситонов в исследованных твердых растворах $\mathrm{GaSe}_{1-x} \mathrm{Te}_{x}$ $(x=0.11,0.15)$ при непрерывном возбуждении светом с $h v_{\text {exc }}>E_{g}\left(E_{g}-\right.$ ширина запрещенной зоны кристалла) приведены на рис. 1. В спектрах доминирует широкая

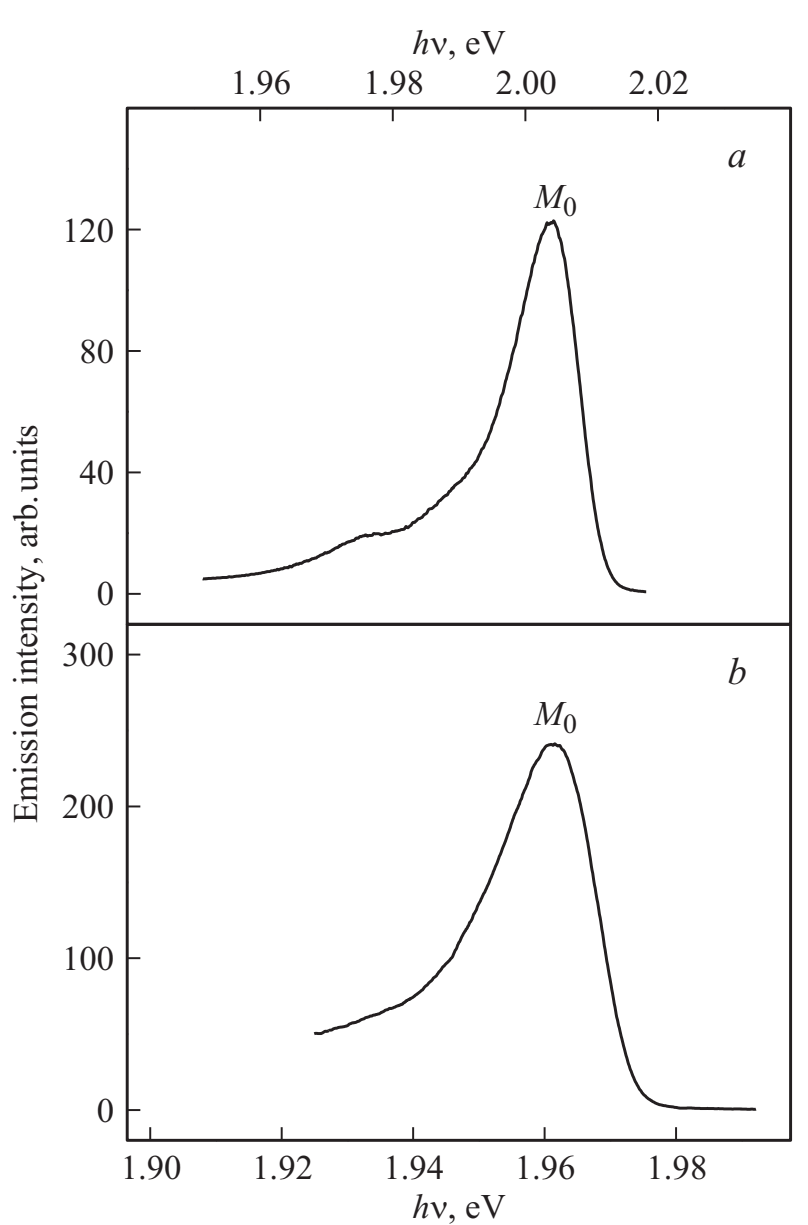

Рис. 1. Спектры фотолюминесценции твердых растворов $\mathrm{GaSe}_{1-x} \mathrm{Te}_{x}$ с $x=0.11(a)$ и $x=0.15$ (b) при межзонном возбуждении. $T=2 \mathrm{~K}$.

асимметричная полоса излучения $M_{0}$, максимум которой смещается в длинноволновую сторону по мере увеличения содержания теллура. На длинноволновом спаде полосы $M_{0}$ в спектре $\mathrm{GaSe}_{0.89} \mathrm{Te}_{0.11}$ заметен слабый максимум излучения, сдвинутый относительно максимума $M_{0}$ на $\sim 0.028 \mathrm{eV}$, что близко к энергии оптического фонона в „чистом“ GaSe [13] и позволяет интерпретировать длинноволновый максимум как колебательный спутник $M_{0}$. Форма полосы фотолюминесценции отражает распределение ЛЭ по состояниям с различной энергией локализации $\varepsilon[4,14]$, при этом положение коротковолнового края приблизительно соответствует энергии ЛЭ на границе подвижности $E_{m}[4]$. При увеличении температуры кристалла интенсивность люминесценции быстро уменьшается. Форма полосы люминесценции, ее спектральное положение и температурная зависимость типичны для ЛЭ.

Спектры возбуждения фотолюминесценции ЛЭ в кристалле $\mathrm{GaSe}_{0.85} \mathrm{Te}_{0.15}$ для различных спектральных участков полосы $M_{0}$, отвечающих ЛЭ с различными энергиями $E_{L E}=E_{m}-\varepsilon$, приведены на рис. 2. (Поскольку спектры возбуждения люминесценции ЛЭ в растворах 
c $x=0.11$ и 0.15 качественно подобны, в дальнейшем мы ограничимся описанием экспериментальных результатов, относящиеся к составу $x=0.15)$. Вид спектров возбуждения существенно зависит от энергии локализации экситонов. Спектр возбуждения состояний с $E_{L E} \approx E_{m}(\varepsilon \approx 0)$, формирующих коротковолновый край полосы излучения на рис. $1\left(h v_{d e t} \geq 1.978 \mathrm{eV}\right)$, содержит одну слабую широкую полосу с максимумом при $h v_{\text {exc }}^{0} \approx 1.99 \mathrm{eV}$. В спектре поглощения раствора этой особенности отвечает полоса экситонного поглощения, которая при $x=0$ соответствует линии поглощения прямого свободного экситона $n=1$ в кристалле GaSe [15]. С увеличением $\varepsilon$ и соответственно уменьшением $h v_{d e t}$ в

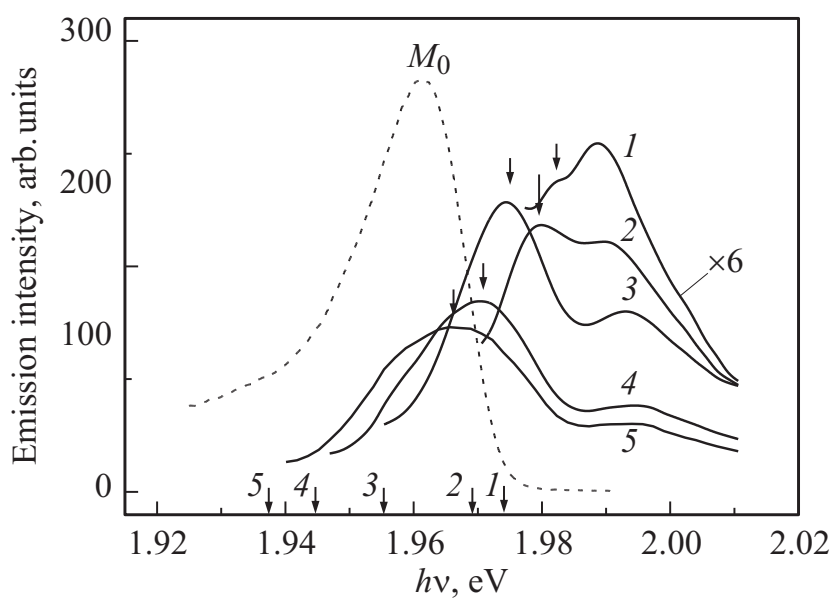

Рис. 2. Спектры возбуждения фотолюминесценции твердого раствора $\mathrm{GaSe}_{0.85} \mathrm{Te}_{0.15}$ (кривые 1-5) для различных энергий детектируемых фотонов $h v_{d e t}$ (величины указаны стрелками 1-5 внизу). Стрелками вблизи кривых отмечены максимумы $M_{E}$ в спектрах возбуждения. Пунктирная кривая представляет спектр излучения ЛЭ в $\mathrm{GaSe}_{0.85} \mathrm{Te}_{0.15}$ при межзонном возбуждении. $T=2 \mathrm{~K}$.

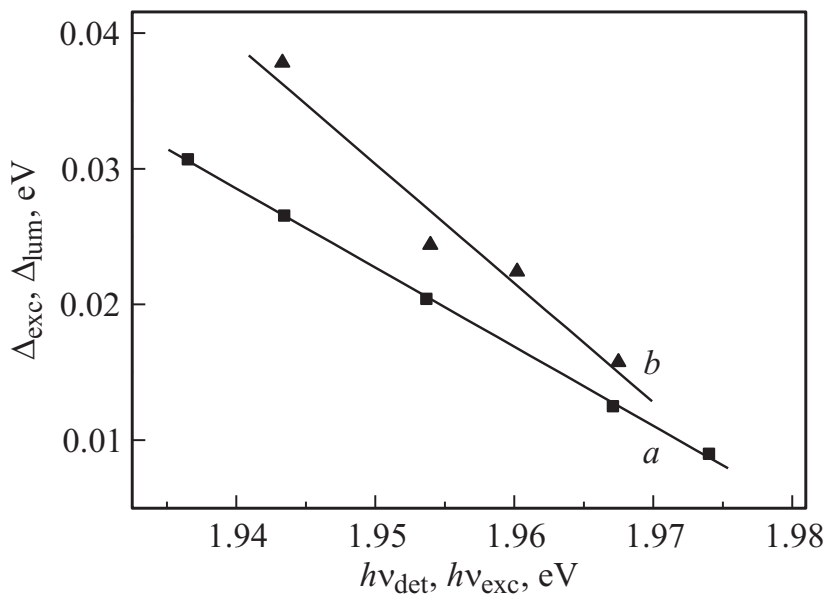

Pис. 3. Зависимости смещений максимумов в спектрах возбуждения фотолюминесценции и излучения твердого раствора $\mathrm{GaSe}_{0.85} \mathrm{Te}_{0.15}, \Delta_{\text {exc }}(a)$ и $\Delta_{\text {lum }}(b)$, от энергий детектируемых $\left(h v_{d e t}\right)$ и возбуждающих $\left(h v_{\text {exc }}\right)$ фотонов соответственно. Точки - экспериментальные значения, отрезки прямых линейная аппроксимация. $T=2 \mathrm{~K}$.

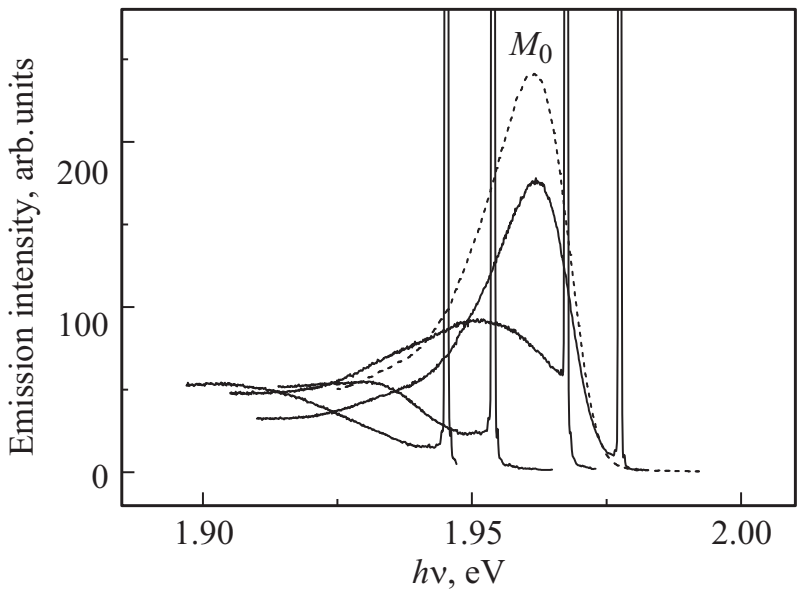

Рис. 4. Спектры фотолюминесценции твердого раствора $\mathrm{GaSe}_{0.85} \mathrm{Te}_{0.15}$ при различных энергиях возбуждающих фотонов. Узкие линии соответствуют спектру возбуждающего лазерного излучения. Пунктирная кривая представляет спектр излучения ЛЭ в $\mathrm{GaSe}_{0.85} \mathrm{Te}_{0.15}$ при межзонном возбуждении. $T=2 \mathrm{~K}$.

спектрах возбуждения возникает дополнительная длинноволновая полоса $M_{E}$ с максимумом при $h v_{e x c}^{M}$, которая по мере уменьшения $h v_{d e t}$ становится доминирующей в спектре (рис. 2). С уменьшением $h v_{d e t}$ полоса смещается в длинноволновую сторону, при этом спектральный интервал между энергией детектируемого фотона $h v_{d e t}$ и положением длинноволнового максимума $h v_{e x c}^{M}$ в спектрах возбуждения увеличивается (рис. 2). Величина расщепления $\Delta_{e x c}=h v_{e x c}^{M}-h v_{d e t}$ увеличивается практически линейно с уменьшением $h v_{d e t}$ (рис. $3, a$ ). В предположении линейной зависимости $\Delta_{e x c}$ обращается в нуль при $h v_{d e t} \approx 1.989 \mathrm{eV}$, то есть в области коротковолновой экситонной полосы в спектре возбуждения.

Для интерпретации спектров возбуждения фотолюминесценции ЛЭ значительный интерес представляет сравнение спектров возбуждения со спектрами фотолюминесценции ЛЭ при их резонансном возбуждении монохроматическим светом с $h v_{\text {exc }}$ из области полосы излучения $M_{0}$ (рис. 1). Полученный в этих условиях спектр фотолюминесценции состоит из широкой полосы $M_{L}$, максимум которой $h v_{\text {lum }}^{M}$ смещен в длинноволновую сторону относительно возбуждающей линии на величину $\Delta_{\text {lum }}$ (рис. 4). Энергетическое расстояние между положением возбуждающей линии $h v_{e x c}$ и положением максимума в спектре люминесценции $\Delta_{l u m}=h v_{\text {exc }}-h v_{\text {lum }}^{M}$ увеличивается практически линейно с уменьшением $h v_{\text {exc }}$ (рис. 3,b). В предположении линейной зависимости $\Delta_{\text {lum }}$ обращается в нуль при $h v_{\text {exc }} \approx 1.985 \mathrm{eV}$.

\section{4. Обсуждение результатов}

Спектры возбуждения фотолюминесценции и спектры фотолюминесценции ЛЭ в исследованных твердых растворах характеризуются рядом общих особенностей: (i) они состоят из широких полос, смещенных в сторону 
более высоких частот по отношению к частоте детектируемого экситонного излучения $h v_{d e t}$ в спектрах возбуждения и в сторону более низких частот относительно энергии возбуждаемого экситонного состояния $h v_{\text {exc }}$ в спектрах фотолюминесценции; (ii) сдвиги положений максимумов полос в спектрах возбуждения и спектрах излучения относительно $h v_{d e t}$ и $h v_{e x c}, \Delta_{e x c}$ и $\Delta_{l u m}$ соответственно увеличиваются по мере уменьшения $h v_{d e t}$ и $h v_{\text {exc. }}$. Если частота $h v_{d e t}$ в спектре возбуждения совпадает с частотой $h v_{\text {exc }}$ в спектре излучения, то $\Delta_{\text {exc }} \cong \Delta_{\text {lum }}$ и, таким образом, положения максимумов в спектре возбуждения фотолюминесценции и спектре излучения характеризуются зеркальной симметрией относительно частоты $h v_{d e t}=h v_{\text {exc }}$ (рис. 5).

Как известно, наличие зеркальной симметрии спектров излучения и поглощения является характерной чертой электронно-колебательных спектров [16,17]. В твердых полупроводниковых растворах с анионным замещением оптические спектры поглощения и излучения, связанные с процессами рождения и аннигиляции ЛЭ, характеризуются сильным неоднородным уширением. В этих условиях правило зеркальной симметрии электронно-колебательных спектров излучения и поглощения, в общем, нарушается [18].

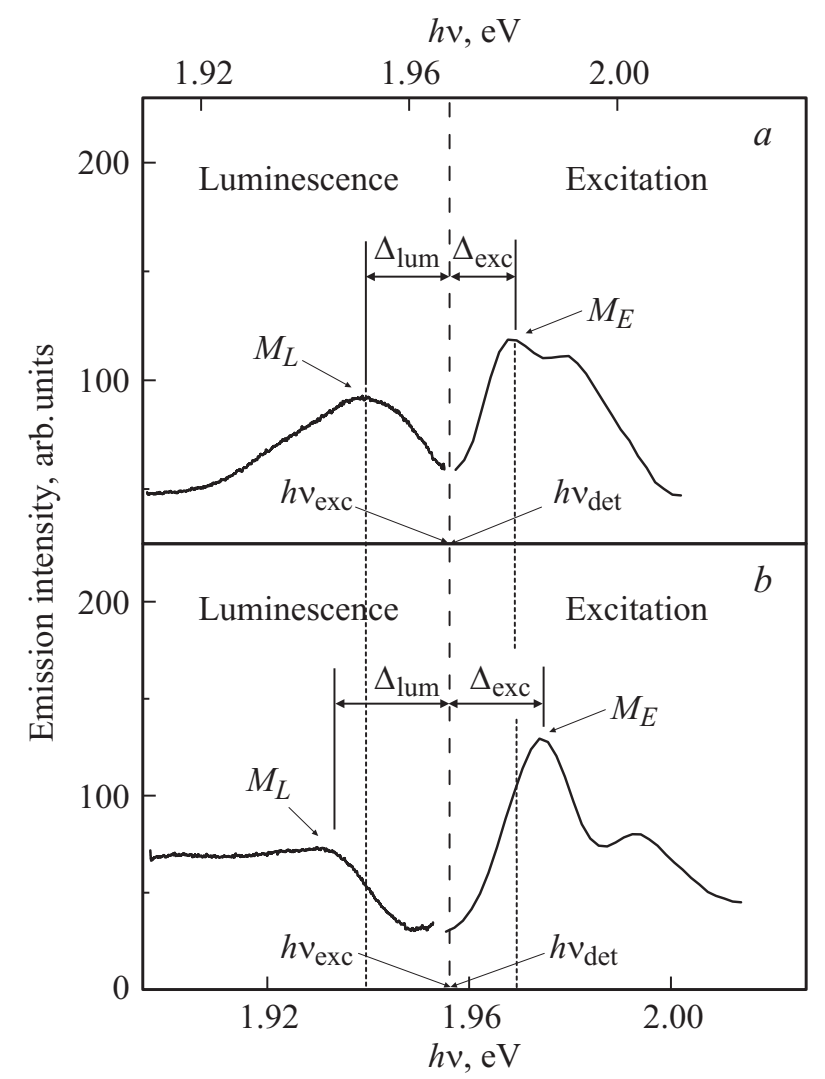

Рис. 5. „Зеркальная“ симметрия положений полос в спектрах фотолюминесценции и возбуждения фотолюминесценции твердого раствора $\mathrm{GaSe}_{0.85} \mathrm{Te}_{0.15}$ для двух различных энергий возбуждающих и детектируемых фотонов $h v_{\text {exc }}=h v_{\text {det }} \approx 1.968 \mathrm{eV} \quad(a)$ и $h v_{\text {exc }}=h v_{d e t} \approx 1.956 \mathrm{eV} \quad(b)$. $T=2 \mathrm{~K}$.
Рассмотрим частный случай, когда неоднородное уширение не играет заметной роли. Пусть имеется система одинаковых излучающих центров (дефектов) в кристалле, которым в нашем случае можно сопоставить набор изолированных друг от друга ЛЭ с одинаковой энергией локализации и одинаковым окружением. При резонансном возбуждении локализованного экситона светом в кристалле рождается электронно-дырочная пара, состоящая из локализованной короткодействующим потенциалом дырки (в случае системы $\mathrm{GaSe}_{1-x} \mathrm{Te}_{x}$ ) и связанного с ней кулоновскими силами электрона. В этом случае, как отмечалось выше, распределение заряда в малой области кристалла (области дефекта) может заметно меняться при переходе центра из основного состояния в возбужденное (отвечающее локализованному экситону). В результате равновесная конфигурация ионов кристаллической решетки в области дефекта становится нестабильной и ионы с течением времени принимают новые положения равновесия, отвечающие минимуму потенциальной энергии $[17,19]$. Соответственно часть энергии электронного возбуждения передается решетке в виде фононов.

Согласно принципу Франка-Кондона процесс решеточной релаксации занимает больше времени в сравнении со временем электронного перехода, так что последний происходит при неизменной конфигурации решетки. Электронный переход из возбужденного в основное состояние дефекта происходит при новой конфигурации решетки. Вслед за излучательным переходом следует перегруппировка ионов в первоначальную конфигурацию, также сопровождаемая рождением фононов. В результате передачи энергии решетке на этапах возбуждения и излучения энергия излучаемых фотонов оказывается меньше энергии возбуждения. Описанный процесс иллюстрирует рис. 6, на котором представлены адиабатические потенциалы дефекта в основном и возбужденном состояниях в зависимости от конфигурационной координаты. Горизонтальными отрезками внутри конфигурационных кривых схематически показаны энергетические уровни, соответствующие различным колебательным состояниям решетки. Если равновесные межъядерные расстояния, соответствующие минимумам конфигурационных кривых основного и возбужденного состояний, не совпадают (рис. 6), доминировать в спектрах возбуждения и излучения будут колебательные спутники бесфононного перехода. Полученные в рамках этой простейшей модели особенности спектров излучения и возбуждения соответствуют особенностям спектров, приведенных на рис. 2 и 4. В этой связи можно предположить, что максимумы в спектрах излучения и возбуждения на рис. 2 и 4 также обусловлены электронно-колебательными переходами. В рамках такого подхода значительная ширина максимумов в спектрах излучения и возбуждения может быть обусловлена электронно-колебательными переходами с участием различных фононов, в том числе акустических. Заметное влияние на форму спектров в твердых растворах ока- 


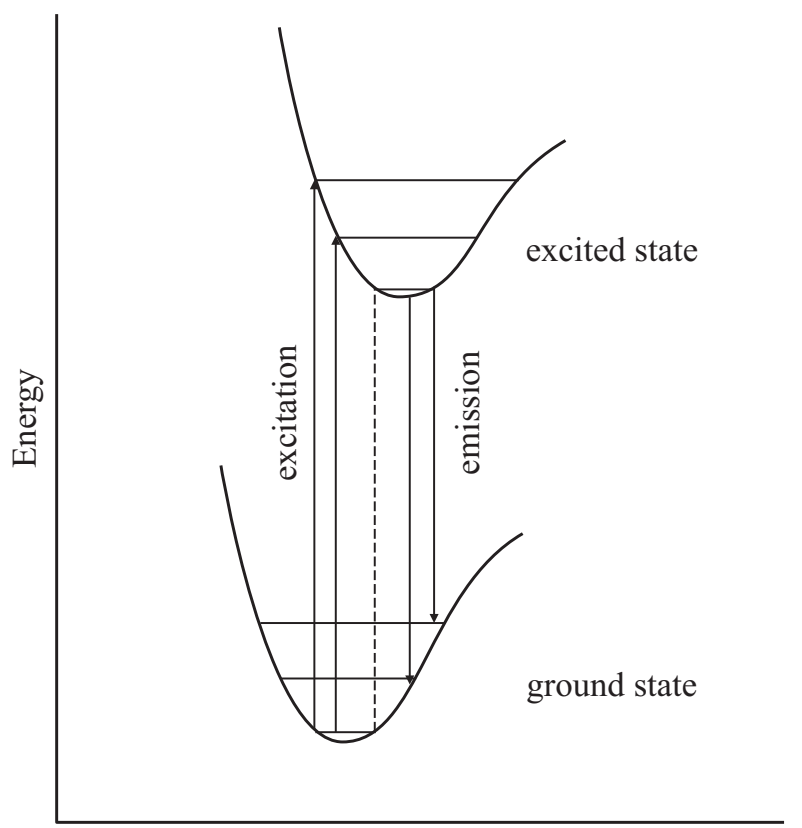

Configurational coordinate

Pис. 6. Адиабатические потенциалы основного и возбужденного (локализованный экситон) состояний дефекта. Вертикальными стрелками отмечены электронно-колебательные переходы с поглощением и излучением фотона. Пунктирная линия соответствует чисто электронным переходам. Горизонтальными линиями схематически показаны энергетические уровни вибронных состояний.

зывает также сильное неоднородное уширение спектров вследствие наличия набора экситонов с различными энергиями локализации. При этом даже в условиях селективного лазерного возбуждения в кристалле ЛЭ с одинаковой энергией локализации, конфигурация их окружения может быть различна.

Как отмечалось выше, особенностью спектров излучения и резонансного возбуждения локализованных экситонов в $\mathrm{GaSe}-\mathrm{GaTe}$ является монотонное увеличение величин $\Delta_{\text {lum }}$ (в спектрах излучения) и $\Delta_{\text {exc }}$ (в спектрах возбуждения) по мере уменьшения $h v_{\text {exc }}$ и $h v_{\text {det }}$ соответственно (рис. 3$) . \mathrm{B} \mathrm{GaSe}_{1-x} \mathrm{Te}_{x}(x<0.3)$ резонансное возбуждение светом локализованного экситона приводит к рождению электронно-дырочной пары, в которой дырка локализована короткодействующим потенциалом „дефекта“, состоящего из кластера атомов теллура. Энергия связи дырки на изоэлектронной ловушке, определяющая, в основном, энергию локализации экситона, весьма чувствительна к изменению конфигурационной координаты: чем больше энергия связи носителя заряда на изоэлектронной ловушке, тем сильнее возмущение решетки [20]. Если аналогичная ситуация имеет место в $\mathrm{GaSe}_{1-x} \mathrm{Te}_{x}(x<0.3)$, уменьшение частоты возбуждающего монохроматического света приводит к возбуждению экситонов с большей энергией локализации, рождение которых связано с более сильным возмущением решетки и, как следствие, с передачей большей части энергии оптического возбуждения фононной подсистеме. В результате Стоксов сдвиг между положениями максимумов в спектрах возбуждения и люминесценции увеличится, что согласуется с экспериментом. Этот эффект, а также возможное различие энергий колебательных квантов решетки в основном и возбужденном состояниях дефекта отчасти объясняют отсутствие идеальной зеркальной симметрии в положениях максимумов в спектрах излучения и возбуждения люминесценции на рис. 5.

Рассмотренная выше модель не рассматривает миграцию энергии между состояниями ЛЭ, которая может заметно влиять на форму спектров излучения и возбуждения. Учет миграции существенен, если вероятность аннигиляции ЛЭ, $W_{R}$, меньше вероятности перехода их в нижележащие состояния с большей энергией локализации, $W_{N R}$. Влияние $W_{N R}$ на спектры излучения локализованных ЛЭ при их резонансном возбуждении в пренебрежении электрон-фононным взаимодействием рассмотрено в [21]. Согласно [21] в условиях резонансного возбуждения ЛЭ монохроматическим светом энергетическое расстояние между положением возбуждающей линии и максимумом в спектре излучения уменьшается по мере уменьшения $h_{e x c}$. Подобное поведение излучения локализованных экситонов экспериментально наблюдалось, в частности, в твердых растворах $\mathrm{CdS}-\mathrm{CdSe}$ [22]. Уменьшение расстояния между возбуждающей линией и максимумом в спектре излучения при уменьшении $h_{\text {exc }}$ не соответствует экспериментальным результатам, приведенным на рис. 3 и 4, что позволяет предположить существенный вклад электрон-фононного взаимодействия в формирование спектров излучения и возбуждения локализованных экситонов в $\mathrm{GaSe}_{1-x} \mathrm{Te}_{x}$ в условиях их резонансного возбуждения.

\section{5. Заключение}

Таким образом, исследование спектров фотолюминесценции и возбуждения фотолюминесценции кристаллов $\mathrm{GaSe}_{1-x} \mathrm{Te}_{x}(x<0.3)$ в условиях резонансного возбуждения ЛЭ позволяет предположить, что широкие полосы в спектрах возбуждения и излучения обусловлены электронно-колебательными переходами с рождением и аннигиляцией ЛЭ соответственно. В рамках модели наблюдаемое поведение спектров указывает на релаксацию решетки, вызванную рождением и аннигиляцией локализованных экситонов, при этом степень возмущения решетки увеличивается с увеличением энергии локализации экситонов. В этой связи представляет интерес выяснение роли слоистой структуры исследованных кристаллов в проявлении указанного эффекта и сравнительное изучение спектров излучения и возбуждения люминесценции локализованных экситонов в слоистых смешанных кристаллах различного элементного состава. 


\section{Список литературы}

[1] S. Adachi. Properties of Semiconductor Alloys: Group-IV, III-V and II-VI Semiconductors. John Wiley \& Sons Ltd., Chichester, U.K. (2009). 400 p.

[2] Дж. Займан. Модели беспорядка. Мир, М. (1982). 592 с.

[3] Ж.И. Алфёров, Е.Л. Портной, А.А. Рогачев. ФТП 2, 1194 (1968).

[4] А.А. Клочихин, С.А. Пермогоров, А.Н. Резницкий. ФТТ 39, 1170 (1997).

[5] S. Lai, M.V. Klein. Phys. Rev. Lett. 44, 1087 (1980).

[6] D. Ouadjaout, Y. Marfaing. Phys. Rev. B 41, 12096 (1990).

[7] S. Permogorov, A. Reznitsky. J. Luminescence 52, 201 (1992).

[8] R. Westphäling, T. Breitkopf, S. Bauer, C. Klingshirn. J. Luminescence 72-74, 980 (1997).

[9] J.J. Hopfield, D.G. Thomas, R.T. Lynch. Phys. Rev. Lett. 17, 312 (1966).

[10] R.A. Faulkner, P.J. Dean. J. Luminescence 1-2, 552 (1970).

[11] А.М. Стоунхэм. Теория дефектов в твердых телах. Мир, M. (1978). T. 1. 569 c.

[12] V. Lemos, F. Cerdeira, L. Gourkov. Solid State Commun. 20, 1101 (1976).

[13] C. Perez Leon, L. Kador, K.R. Allakhverdiev, T. Baykara, A.A. Kaya. J. Appl. Phys. 98, 103103 (2005).

[14] А.Г. Абдукадыров, С.Д. Барановский, С.Ю. Вербин, Е.Л. Ивченко, А.Ю. Наумов, А.Н. Резницкий. ЖЭТФ 98, 2056 (1990).

[15] J. Camassel, P. Merle, H. Mathieu, A. Gouskov. Phys. Rev. B 19, 1060 (1979).

[16] J.R. Lakowicz. Principles of fluorescence spectroscopy. 3rd ed. Springer Science+Business Media, LLC, N. Y. (2006).

[17] К.К. Ребане. Элементарная теория колебательной структуры спектров примесных центров кристаллов. Наука. М. (1968). $233 \mathrm{c}$.

[18] Zero-phonon lines and spectral hole birning in spectroscopy and photochemistry / Ed. O. Sild, K. Haller. Springer-Verlag. Berlin Heidelberg (1988). 183 p.

[19] О. Маделунг. Физика твердого тела. Локализованные состояния. Наука. М. (1985). 184 с.

[20] P.J. Dean. J. Luminescence 1, 2, 398 (1970).

[21] M. Oueslati, C. Benoit à la Guillaume, M. Zouaghi. Phys. Rev. B 37, 3037 (1988).

[22] E. Cohen, M.D. Sturge. Phys. Rev. B 25, 3828 (1982). 